SULLA BARITINA DI CALAFURIA; NOTA DEL DOTT. ALCESTE DELLA VALLE .

Alla distanza di cinque miglia circa da Livorno, tenendo la strada regia Maremmana, trovasi lungo la costa, battuta dal mare, una torre chiamata di Calafuria. Essa è costruita sopra il macigno, che, insieme al calcare nummulitico e all'alberese, costituisce i poggi che si estendono fin presso il Romito, quasi sempre scendendo con scabroso declivio insino al mare, ed è in vicinanza delia torre che il solfato di barite trovasi tanto sulla destra che sulla sinistra della strada regia, la quale taglia essi poggi, colà esclusivamente formati di macigno. Il minerale è in vene e filoncelli incassati nella roccia e associato ad altre sostanze.

\title{
Caratteri .
}

Il suo peso specifico è assai variabile, ch' ora vi riscontriamo il 4,3 , ora il 4,4 , ora il 4,6 , ed il 3,6 perfino. La differenza è in relazione colla maggiore o minore purezza dei cristalli ed in special modo colla diversa natura delle sostanze incluse. Non cosi incerla però ne è la durezza, giacchè esperimentandola sopra varj campioni vidi che costantemente mi rappresentava il 3,5 , numero in generale assegnato dagli autori alla barilina, e che credo abbastanza esatto per attribuirlo anche a questa di Calafuria, atlesochè segna energicamente la calcite ed è con eguale intensilà graffiata dalla fluorina . 
Circa alla cristallizzazione la forma ordinaria è quella più o meno tabulare (Tav. II. fig. 1) e riferibile al prisma romboidale dritto con angolo di $101^{\circ} .42^{\prime}$. Oltre a questa, non modificata da alcuna faccia ho avuto occasione di studiare più cristalli, che sull'angolo A preseutavano una serie di faccette appartenenti a macrodomi ottusissimi ed in tal numero da rendere curvi gli spigoli laterali che al suddetto angolo concorrono (fig. 2 ). E osservando certi uni di questi cristalii traverso alla base $P$ si scorge una linea paralella a ciascuno spigolo $\mathrm{B}$; la quale a prima vista potrebbesi prendere come fuccetta di modificazione, se non che attentamente esaminata si riconosce non provenire che da uno strato esterno più 0 meno sottile 0 più 0 meno trasparente, ed alle volte anche opaco, forse determinato da un piano di clivaggio ( $f g .3$ ). Un caso analogo è presentato da molte baritine di altre localita, ad esempio quella di Kapnick, come lo accenna il Levy, ma mentre in queste ultime distinguonsi bene esse linee esclusivamente derivanti da piani di clivaggio, in quella di Calafuria invece spesso limitano sostanze incluse, vè mi è punto occorso di riscontrarle in cristalli diafani e limpidi, ma sempre in quelli di un color brano e alquanto opachi. Dimandando a me stesso come siansi formati i piani limitati dalle linee in discorso, non ho potuto che supporre essersi complelati $i$ cristalli in tempi distinti, concorrendo dapprima, a modo d'esempio alla formazione loro un solfato diverso per le sostanze estranee, che meccanicamente teneva, da quello che in seguito venne ad accrescerli e che formò all'esterno un nuovo strato dissimile dalle rimanenti parti interne. E questi cristalli, nei quali trovansi inclusi corpi stranieri, sono in generale semplicissimi ed anche se portano qualche modifcazione risultano meno complicati di quella non sieno i più limpidi.

Oltre alla serie dei macrodomi, che modificano l'angolo A, ho ritrovato molli cristalli troncati sullo stesso angolo da una $o$ due sole faccette ben distinte e piane, vale a dire da un solo o due macrodomi, quali sopo indicati dalla (fig. 4.) Altri cristalli, e sono appunto i più limpidi, oltre alla suddetta modifica. zione ne presentano altra sugli spigoli laterali $B$, costituita da faccette di troncatura $(\mathrm{fg} .5$ ) faccelte molto diversamente svilup- 
108

pate e tali anche da scomparire alle volte, onde avviene di vedere, per esempio, troncato uno degli spigoli laterali inferiori $B$, mentre manea ugual moditicazione sul corrispondente superiore ( $f g .6$ ).

Il brachidomo pure non è affatto escluso da questa baritina e ne fa fede qualche raro esemplare ( $f g .7$ ).

Ben limitate, come ognun vede, sono le forme della baritina di Calafuria, ed ho ragione di credere non trovarsene altre diverse dalle descrilte, poichè molti e molti esemplari ho avuto fra mano e per quanto accuritamente gli abbia studiati non ho potuto trovare che la ripetizione delle menzionate forme.

Circa alla grossezza dei cristalli di baritina nulla v' è di fisso, che da quelli minutissimi, da occorrere la lente a distinguerli, si passa gradatamente a quelli di tre a quattro centimetri.

Volendo ora parlare del modo di presentarsi di questi cristalli mi convien dire esser sempre assuciati, spesso compenetrati e ammassati fra loro. Ma se ben si osservano in generale riscontransi impiantati sulla roccia per le facce laterali (com è ordinario a questa sprecie minerale) ed alle vulte in serie fra loro allineate, come lo mostral la (fig. 8). Splesso anche si compenetrano couservando parilleli i loro spigoli, cosi che esamimando l'insteme ci si offruno dei belli e nelti angoli rientranti. Se aviene poi che si disgiumeano due eristalli, che si compeuetravano, uel puito dell antico loro altacco ci si offrono delle strie parallele alle linee di congiungimento a guisa di tramoguia, la quile in certi casi (fig. 9) sembra si manifesti, specialmente quando riscontrasi sulla base $\mathbf{P}$, che potrebbesi prendere per particolare cristallizzazione, se l'ubliquità delle strie, rispetto agli spişoli laterali, e gli anguli rientranti che determinano non stessiero a palesare l'origine delle strie e della supposta tramoggia, come determinata da due cristalli che si cumpenetravano e che stavano adagiati sulla base del cristallo simulante la tremia . Le strie sarebbe inutile dire che stauno ad accennare il successivo accrescersi dei varj cristalli. 
Associazioni.

Come dicevo in principio la baritina, che ho preso a descrivere, sti spesso associata al altre sostanze minerali, tali sono il ruarzo il sesquiossido di ferro, la dolomite ferruginosa ec. In gencrale i suoi cristalli si presentano opachi e più o meno colorati a causa del ferro che frequentemente li sporca. Ninn per questo peri non esistono dei cristalli limpidisimi, benrlhe rari assai pur ne posseggo, ed è qui ad accennare averli sempre rilrovati associati e frammisti a piccoli e bei crislallini di dolomile ferruginosa, la quale darebbe a supporre di essersi assorhito tulto il ferro, lascianilone così perfellameute libero il sulfato di barile. E già che cade in acconcio non posso passare innanzi senza spendere una parola anche per questa dolomite, la quale, se non fosse che per il solo suo vago aspetto, presenta tanto di interessunte da meritar di parlarne.

Trovasi in soltili venuze incluse nel macigno, tanto associata ai filoncelli rli barilina, che affalto snla. I suoi cristalli slauno a rappresentare dei piecoli romboedri a facce curve, ed anche aniloghi a quelli ofterlici dalla cosi detla dolomite linwuiforme, ma anzichè presentare lo splendore madreperlaceo di quest ultıma, essa tronasi quasi sempre rivestita di sesquiossido di ferro, non sempre però di eguale aspetto, che ora è scura e opaca, ora lucente e più russigna, ora quasi chiara ed iridescente, ed e speciainente al rontatto della scura ed opaca che la barilina si moitra diafana e limpida.

Il quarzo è altro mincrale che si associa alla barilina, frammischiaudosi i loro cristilli. Esso, al pari della dolomite, costituisce per se solo dei filonrelli nel inacigıo; quando però àsociato al solfato dalla disponizione dei suoi crislalli facilmente si riconosce anteriore alla formazione di quelli di barite, giache questi ultimi non di rido trovansi impiantati sulle futece di quelli di quarzo, ma oltre a cir spesso le pareti dei filonelli souo tappezate di quarzo eristallizato, mentre la baritina ne riempie la parte mudiana. Lo slesso non si pinò dire della dolomite, la quale tutte le volte che trovasi asso- 
ciata al solfato di barite si addossa a questo con i suoi cristallini, attestando invece posteriorita di origine.

Quel sesquiossido di ferro, che ricuopre la dolomite, in certi esemplari riveste totalmente anche il solfato di barite e cio riscontrasi particolarmente in alcuni filoncelli, nei quali è caso trovare cristalli limpidi e liberi della suindicata veste. Nè ciò fa maraviglia, perchè il macigno di Calafuria è talmente compenetrato dal ferro da appalesarci alla sua superficie, ove questo è maggiormente concentralo, come una rete di venuzze rilevate, le quali al certo ci si mostrano tali per aver resistito più lungamente all' azione degli agenti esterni di quello non lo abbia l'arenaria includente. Ora può forse supporsi che le acque pluviali, che lavano essa roccia, sciolgano porzione di quel ferro, e, trasportandolo seco, lo depositino entro i floncelli e sui cristalli dei diversi minerali,

Ho sopra accennato che i cristalli di barilina ordinariamente tengono incluse sostanze straniere. Il sesquiossido di ferru, se non li riveste li colora, che rarissimi, come ho pur notato, sono quelli del tutto limpidi. Mi altro minerale cristallizzato, che non era stato avvertito a Calafuria, e che l' analisi mi ha fatto riconoscere di antimonio (stibina) con varia disposizione si appalesa entro ai cristalli di baritina, cosicchè ora vi si vedono dei flamenti isolati e di splendore metallico, ora confusi e frammisti, terminando spesso nell' insieme a linee alquanto discoste e parallele agli spigoli dei cristalli stessi, (fg. 10). Altra volta essi flamenli si dipartono in fascio irraggiante da una delle linee suddette ( $f g .11)$ altre invece da un punto interno, costituendo allora raggi distinti (fig. 12 e 13). I cristalli di stibina, oltre al trovarsi inclusi in quelli di baritina, spesso con le loro estremita sporgono al di fuori delle facce. La disposizione fibrosa raggiata di essa stibina non solamente si riscontra nei cristalli, ma anche nella massa compatla che costituisce il filoncello di barite.

Questo minerale di antimonio si riconosce spesso che ha subito un' alterazione e si è decomposto; infatli molli cristalli di baritina son tutti perforati da canaletti di forma romboidalt, alcuni dei quali vuoti, altri pieni di una sostanza giallorossastra, analoga a quella che nello spessor dei flloncelli or- 
dinariamente stà associata alle concentrazioni di stibina, appalesando la successione di questa al kermes e forse poi alla cervantite. E che questi ultimi minerali vi esistano me ne sono accertato analizzando la sostanza giallo-rossastra, la qualo mi ha con chiarezza mostrato risultare prevalentemente di antimonio. Qui giova notare come i cristalli di barite, che includono la stibina, sieno quelli appunto che somministrano un peso specifico minore, circa 3,6 , fatto abbastanza strano sapendo che la slibina ha un peso specifico maggiore della baritina, ma facilmente spiegabile se si riflette che possibilmente alcuni dei cristalli di stibina si saran decomposti e avran lasciato nell' interno dei canaletli vuoti .

\section{Giacitura .}

L' arenaria macigno di Calafuria alla superficie è fratturata, mostrando crepe in tulti i sensi quasi che fosser piani di ritiro, ed $\grave{e}$ in molte di esse che stanno le concentrazioni di sesquiossido di ferro, di quarzo, di solfaţo di barite ec. Riguardo alla direzione delle vene di baritina potrebbesi dire non averne alcuna, a causa delle tante che ci presentano. Forse le renuzze, che si appalesano alla superficie, concorrono almeno nell' interno, ad una cerla profundila ad un filone unico, ma la mancanza di profondi scavi mi ha tolla la possibilità di formare un giudizio, però, dalle ripetute osservazioni intorno alla direzione delle vene, nell'insieme ho poluto riscontrarne molte parallele e dire!te circa da E. ad 0 . ed altre pur parallele, ma normali alle precedenti, cioè volte da N. e S. In generale la direzione è oscillante fra il N. c l'o. al S. e l'E.

Sembra quindi aversi in questa giacitura indizio di una di quelle dighe quarzoso melallifere generalmente diretle $\mathbf{N}$. S. ed accompagnate da filoni e vene normali a quella direzione, che sono tanto frequenti nella Maremma. E per la presenza dell' antimonio potrebbesi questa nostra paragonare a quella di Prata nel Masselano, di Pereta e di Monteauto, ma da esse tutte è diversa per la presenza della barilina. 